\title{
A hybrid adaptive unscented Kalman filter algorithm
}

\author{
Jun He, Yong Chen, Zhaoxia Zhang, Wentao Yin, Danfeng Chen \\ School of Automation, Foshan University, Foshan 528000, CHINA \\ e-mail: hejun_723@fosu.edu.cn
}

\section{SUMMARY}

In order to overcome the limitation of the traditional adaptive Unscented Kalman Filtering (UKF) algorithm in noise covariance estimation for state and measurement, we propose a hybrid adaptive UKF algorithm based on combining Maximum a posteriori (MAP) criterion and Maximum likelihood (ML) criterion, in this paper. First, to prevent the actual noise covariance deviating from the true value which can lead to the state estimation error and arouse the filtering divergence, a real-time covariance matrices estimation algorithm based on hybrid MAP and ML is proposed for obtaining the statement and measurement noises covariance, respectively; and then, a balance equation the two kinds of covariance matrix is structured in this proposed to minimize the statement estimation error. Compared with the UKF based MAP and based ML, the proposed algorithm provides better convergence and stability.

KEY WORDS: $\quad$ hybrid adaptive; unscented Kalman filtering; maximum a posteriori; maximum likelihood criterion.

\section{INTRODUCTION}

In the state estimation techniques for linear stochastic systems, the Kalman filter has received many attention industrial areas widely and achieved some progress [1]. However, in real application, it is difficult to obtain exact unknown noise statistics information which may induce large state estimation errors and filter divergence. To make up for this defect, a number of modified filter methods have been published to weaken the impact of uncertainly noise information, and so the adaptation of noise covariance matrices has become a important direction for developing stability and convergence Kalman fiter [2].

Kinds of extend Kalman filter (EKF) have been proposed for solving the nonlinear problem in stochastic systems. EKF is the first extend of the KF in historically, which approximates the state parameter for nonlinear system by its first order linearization [3-4]. Although it is simple and easy to use for nonlinear state estimation, but its prominent defects, the non-Gaussian of nonlinear system state distributions, have not been taken into count and the deviation of estimation mean and covariance for state parameter have been shamefully neglected. Moreover, as the sample number growing the estimation errors resulting by the linearization of Kalman filter can accumulate [5-6]. In order to reduce the constraints of the first-order 
Taylor expansion of EKF naturally, as reported in [7-8], a novel adaptive robust filter method which can approximate a probability distribution than to approximate an arbitrary nonlinear transformation, named unscented Kalman filter (UKF), has been proposed for state estimation of nonlinear stochastic systems. The natural advantage of UKF is the third-order Taylor expansion approximate used to obtain the conditional mean and variance for Gaussian noises accurately, which does not rely on any linearization processing for state predictions and covariance. Consequently, compared with the robust EKF, the UKF has been used in numerous industrial applications, such as state estimation and remaining useful life predict for rotary machines [9-11], battery health management [12-13], target tracking and satellite-aided navigation [14-15], among others. However, in real industrial application, the unknown process noise is frequency encountered, and the accumulated state estimation errors, in the case of the process noise covariance is not obtained accurately, become more and more serious.

To overcome these unfavorable factors, many researchers have paid their attentions to two important directions to improve the stability and convergence of UKF, and some works have published in the two mainly purposes, the one is how to improve the matching degree of noise distribution obtained by UKF and from a real stochastic system; the other is how to adjust the estimation window for the UKF which the covariance matrix of uncertainly noise is estimated at the present time. To solve the problem that the inaccuracy and divergence of UKF when the prior noise statistic is unknown, an adaptive UKF algorithm based on maximizing a posterior estimation and exponential weighting was proposed by authors [16]. In order to solve the state estimation problem of nonlinear systems without knowing prior noise statistical characteristics, an adaptive UKF based on the maximum likelihood principle and expectation maximization algorithm was proposed [17]. Deng.et al in [18] proposed an adaptive unscented Kalman filter (AUKF) to estimate the time-varying parameters and states of a kind of nonlinear high-speed objects, which a strong tracking filter and wavelet transform were employed to improve the robustness of unscented Kalman filter (UKF) under the process noise is inaccuracy, and the estimate accuracy by the variance of measurement noise, respectively. In [19], an adaptive unscented Kalman filter algorithm with dynamic thresholds of covariance used to update measurement noise covariance matrix real-time was developed for satellite fault detection and diagnosis by the authors. As reported in [20], an adaptive unscented Kalman filter method with using adaptive error covariance matching technology to improve the state estimation accuracy was employed to estimate the battery energy and power capability by authors. A robust Masreliez-Martin UKF which can provide reliable state estimates in the presence of both unknown process noise and measurement noise covariance matrices was presented [21]. In the estimation process, an adaptive adjustment of the noise covariance using covariance matching in the UKF was implemented by authors [12], which was used to get a more accurate and robust state of charge estimation. Meanwhile, Estimation window, is one of the important factor which can make the stability and accuracy of UKF be effected, has been concerned by the academic community, recently [22-23]. These windowing approaches were adopted to estimate the unknown noise covariance matrix based on a set of innovation sequences in the window, instead of predicting the noise covariance matrix by the historical innovation sequences.

From the review of the related work mentioned above, it can be found that, though there are some reports focusing on robust of adaptive UKF method or windowing approaches adopted to estimate the unknown noise covariance matrix, separately, few works concentrate on the adjustment of matching degree between noise estimation covariance obtained by UKF and the 
truth value from real stochastic system, adaptively. As stated before, a hybrid adaptive method helps to make the unknown noise estimation covariance computed by UKF match the truth value of real stochastic system as much as possible. The hybrid adaptive UKF method based on MAP and ML criterion for nonlinear stochastic system state estimation contributes to improve the stability and convergence of state estimation based on UKF. Accordingly, in this paper, there are three major contributions. First, we propose a hybrid adaptive UKF method for uncertainly noise covariance estimation of a nonlinear stochastic system, and utilize the MAP method to obtain a suboptimal and unbiased estimation for slacking down the impact of the mendacious and deviation for state estimation. Second the maximum likelihood principle is used to obtain unknown noise estimation covariance for real nonlinear stochastic system, no matter the MAP method and ML method, there lies deviation of estimation covariance and truth value. Last, the unknown noise covariance is estimated by the two algorithms (MAP and $M L)$, respectively, and then the two covariance are performed the expectation of their sum, which looked as the new unknown noise estimation covariance, and the new stability discriminator rule of UKF algorithm is employed.

\section{UNSCENTED KALMAN FILTER}

Given a nonlinear stochastic system which described as follows [24]:

$$
\begin{gathered}
x_{k}=f\left(x_{k-1}\right)+q_{k-1} \\
y_{k}=h\left(x_{k}\right)+r_{k}
\end{gathered}
$$

where $x_{k} \in \mathfrak{R}^{n}$ is the state parameter, $y_{k} \in \mathfrak{R}^{m}$ represents the measurement parameter, $q_{k-1}$ and $r_{k}$ indicate the process noise and measurement noise, which can meet the condition of zero mean and covariance being defined as follows, respectively:

$$
E\left[q_{k-1} q_{k-1}^{T}\right]=Q_{k-1}, E\left[r_{k} r_{k}^{T}\right]=R_{k}
$$

All the UKF variants, as in the EKF, keep the structure of the Kalman filter for linear system. There are two steps for compute the UKF, which including one prediction (or a priori estimation) and one correction (or update) likes KF.

A) Predict: Given a set of $2 n+1$ sigma points which derived from estimated state, described as blows:

$$
\begin{aligned}
& \chi_{k-1 / k-1}^{0}=\hat{x}_{k-1 / k-1}, \\
& \chi_{k-1 / k-1}^{i}=\hat{x}_{k-1 / k-1}+\left(\sqrt{(n+\lambda) P_{k-1 / k-1}}\right)_{i}, i=1, \cdots, n \\
& \chi_{k-1 / k-1}^{i}=\hat{x}_{k-1 / k-1}-\left(\sqrt{(n+\lambda) P_{k-1 / k-1}}\right)_{i}, i=n+1, \cdots, 2 n
\end{aligned}
$$

where $\left(\sqrt{(n+\lambda) P_{k-1 / k-1}}\right)_{i}$ is the $i$ th row/column of the matrix square root of $(n+\lambda) P_{k-1 / k-1}$ for the $k$ th time step.

Then the predicted sigma points propagated through the transition function $f(\cdot)$, and the prior of these predicted sigma points namely mean, and covariance are computed by: 


$$
\begin{aligned}
& \chi_{k / k-1}^{i}=f\left(\chi_{k-1 / k-1}^{i}\right), i=0, \cdots, 2 n \\
& \hat{x}_{k \mid k-1}=\sum_{i=0}^{2 n} W_{m}^{i} \chi_{k / k-1}^{i}, \\
& P_{k-1 / k-1}=\sum_{i=0}^{2 n} W_{c}^{i}\left(\chi_{k \mid k-1}^{i}-\hat{x}_{k / k-1}\right)\left(\chi_{k \mid k-1}^{i}-\hat{x}_{k / k-1}\right)^{T}+Q_{k-1}
\end{aligned}
$$

where $W_{m}^{i}$ represents the weight of $i$ th sigma point state mean, $W_{c}^{i}$ is the weight of $i$ th covariance estimation, which given as follows, respectively:

$$
\begin{aligned}
& W_{m}^{0}=\frac{1}{n+\lambda} \\
& W_{c}^{0}=W_{m}^{0}+\left(1-\alpha^{2}+\beta\right) \\
& W_{c}^{i}=W_{m}^{i}=\frac{1}{2(n+\lambda)}, i=1, \cdots, 2 n
\end{aligned}
$$

Scaling factor $\lambda$ is defined as:

$$
\lambda=\alpha^{2}(n+\kappa)-n
$$

where $\alpha$ and $\kappa$ were the scaling factor used to control the spread of the sigma points, $\beta$ describes the related distribution of $x_{k}$.

B) Update: As well as in the predict step above, given a set of $2 n+1$ sigma points, which used to compute the filtered mean and covariance:

$$
\begin{aligned}
& \chi_{k / k-1}^{0}=\hat{x}_{k / k-1}, \\
& \chi_{k / k-1}^{i}=\hat{x}_{k / k-1}+\left(\sqrt{(n+\lambda) P_{k / k-1}}\right)_{i}, i=1, \cdots, n \\
& \chi_{k / k-1}^{i}=\hat{x}_{k / k-1}-\left(\sqrt{(n+\lambda) P_{k / k-1}}\right)_{i}, i=n+1, \cdots, 2 n
\end{aligned}
$$

And then, the observation function was used to project these sigma points, which given as follows:

$$
Y_{k}^{i}=h\left(\chi_{k / k-1}^{i}\right), i=0, \cdots, 2 n
$$

Then, the predicted measurement mean and covariance were obtained by recombined the weighted sigma points, and the state measurement cross-covariance matrix, all that can be written as follows:

$$
\begin{gathered}
\hat{y}_{k}=\sum_{i=0}^{2 n} W_{m}^{i} Y_{k}^{i} \\
P_{z Z}=\sum_{i=0}^{2 n} W_{c}^{i}\left(Y_{k}^{i}-\hat{y}_{k}\right)\left(Y_{k}^{i}-\hat{y}_{k}\right)^{T}+R_{k} \\
P_{X Z}=\sum_{i=0}^{2 n} W_{c}^{i}\left(\chi_{k \mid k-1}^{i}-\hat{x}_{k \mid k-1}\right)\left(Y_{k}^{i}-\hat{y}_{k}\right)^{T}
\end{gathered}
$$

Equation (11) and Eq. (12) were used to compute the gain of UKF, described as follows:

$$
G_{k}=P_{X Z} P_{z Z}^{-1}
$$


And then, the updated state and updated covariance can be computed with these formulate described as:

$$
\begin{gathered}
\hat{x}_{k / k}=\hat{x}_{k / k-1}+G_{k}\left(y_{k}-\hat{y}_{k}\right) \\
P_{k / k}=P_{k / k-1}-G_{k} P_{z z} G_{k}{ }^{T}
\end{gathered}
$$

\section{ADAPTIVE UKF}

In this section, a hybrid adaptive UKF will be introduced, which incorporates the UKF based on MAP and the UKF based on ML, respectively. Following, we will introduce the UKF based on MAP, the UKF based on ML criterion and hybrid adaptive UKF, respectively.

\subsection{MAP CRITERION}

There are two mainly factors which can lead to the filter divergence, one is the unreasonable model for a stochastic system, and the other one is inaccurate noise statistical characteristics, which have attracted many attentions in how to solve two problems adaptive. MAP, a classical adaptive statistical method, employed to develop a suboptimal noise estimator by Sage and Husa in 1960s [25].

The estimators $\hat{Q}_{k}$ and $\hat{R}_{k}$ based MAP for $Q$ and $R$ at the $k$ step will be obtained such as to maximum the a-posteriori density function, which described as:

$$
p[x(k), Q, R / y(k)]=\frac{p[y(k) \mid x(k), Q, R] p[x(k), Q, R]}{p[y(k)]}
$$

Because of $y(k)$ is independent of the parameters $x(k), Q, R$. Furthermore, the state parameter and the covariance of measurement noise are assumed to be independent mutually, so that, the estimator based on MAP may be obtained by maximization the unconditional density function, and then the Eq. (16) rewritten as follows:

$$
J(k)=p[y(k) / x(k), Q, R] \times p[x(k)] p[Q] p[R]
$$

The prior statistics for $Q$ and $R$ were introduced detailed by Sage and Husa. So, the density functions $p[x(k)]$ and $p[y(k) / x(k), Q, R]$ were:

$$
\begin{aligned}
& p[x(k)]=p[x(0)] \prod_{i=1}^{k} p[x(i) / x(i-1), Q]= \\
& =|p[0 / 0]|^{-\frac{1}{2}}|Q|^{-\frac{k}{2}} \frac{1}{(\sqrt{2 \pi})^{k n+1}} \times \\
& \times \exp \left\{-\frac{1}{2}\left[\sum_{i=1}^{k}\|x(i)-f(x(i-1))\|_{Q^{-1}}^{2}-\|x(0)-\hat{x}(0 / 0)\|_{P^{-1}(0 / 0)}^{2}\right]\right\} \\
& p[y(k) \mid x(k), Q, R]=\prod_{i=1}^{k} p[y(i) \mid x(i), R]= \\
& =\frac{1}{\sqrt{(2 \pi)^{k m}|R|^{k}}} \exp \left\{-\frac{1}{2} \sum_{i=1}^{k}\|y(k)-h(x(i))\|_{R^{-1}}^{2}\right\}
\end{aligned}
$$


where $n$ and $m$ are the dimensions of state parameter and measurement parameter, respectively. And then, combining the density function given in Eq. (17), Eq. (18) and Eq. (19), under the maximization of $J(k)$, the $\hat{Q}_{k}$ and $\hat{R}_{k}$ given as:

$$
\begin{gathered}
\hat{Q}_{k}=\frac{1}{k} \sum_{i=1}^{k}\left[\hat{x}_{i / k}-f\left(\hat{x}_{i-1 / k}\right)\right]\left[\hat{x}_{i / k}-f\left(\hat{x}_{i-1 / k}\right)\right]^{T} \\
\hat{R}_{k}=\frac{1}{k}\left[y_{i}-h\left(\hat{x}_{i / k}\right)\right]\left[y_{i}-h\left(\hat{x}_{i / k}\right)\right]^{T}
\end{gathered}
$$

Although Sage and Hussas' method has many advantages compared with conventional filter methods, such as real-time, simple, et, its stability drop sharp under the unknown process noise and measurement noise. Aiming at this problem, adaptive UKF Filtering algorithm based on maximum a posterior estimation and exponential weighting was published [16], in which the fading factor was introduced to substitute the weighting coefficient, therefore, the noise statistics estimator based on fading factor defined as:

$$
\begin{gathered}
\hat{q}_{k}=\left(1-d_{k}\right) \hat{q}_{k-1}+d_{k}\left[\hat{x}_{k}-\sum_{i=0}^{2 n} W_{m}^{i} f\left(\chi_{k / k-1}^{i}\right)\right] \\
\hat{r}_{k}=\left(1-d_{k}\right) \hat{r}_{k-1}+d_{k}\left[\hat{y}_{k}-\sum_{i=0}^{2 n} W_{m}^{i} h\left(\chi_{k / k-1}^{i}\right)\right] \\
\hat{R}_{k}^{M A P}=\left(1-d_{k}\right) \hat{R}_{k-1}^{M A P}+d_{k}\left[\varepsilon_{k} \varepsilon_{k}^{T}-\sum_{i=0}^{2 n} W_{c}^{i}\left(\chi_{k / k-1}^{i}-\hat{y}_{k / k-1}\right)\left(\chi_{k / k-1}^{i}-\hat{y}_{k / k-1}\right)^{T}\right] \\
\hat{Q}_{k}^{M A P}=\left(1-d_{k}\right) \hat{Q}_{k}^{M A P}+d_{k}\left[G_{k} \varepsilon_{k} \varepsilon_{k}^{T} G_{k}^{T}+P_{k / k-}\right. \\
\left.-\sum_{i=0}^{2 n} W_{c}^{i}\left(\chi_{k / k-1}^{i}-\hat{x}_{k / k-1}\right)\left(\chi_{k / k-1}^{i}-\hat{x}_{k / k-1}\right)^{T}\right]
\end{gathered}
$$

where $\varepsilon_{k}$ is the residual of measurement parameter, which can be computed by $\varepsilon_{k}=y_{k}-\hat{y}_{k / k-1}$, and $i=1, \cdots, 2 n$. The coefficient $d_{k}$ was written as below:

$$
\left\{\begin{array}{l}
\beta_{i}=d_{k} b^{i-1}, i=1, \cdots, n \\
d_{k}=\frac{1-b}{1-b^{k}}
\end{array}\right.
$$

where $b$ was the fading factor, which satisfied $0<b<1$, and $\beta_{i}$ was the $i$ th weighting factor, which defined as $\beta_{i}=\beta_{i-1} b$ and satisfied $\sum_{i=1}^{k} \beta_{i}=1$.

Real time estimation and correction for the unknown time-varying noise statistics carried out with the noise statistics estimator, which may overcome the shortcomings of the traditional UKF in the noise statistics of unknown time-varying filter. However, once the amplitude of real time estimation and correction was not controlled well, which may result in deviation of noise estimation and its theoretical value. In order to make up for this defects, the ML principle and EM criterion were used to employ an adaptive UKF Algorithm [17]. 


\subsection{CRITERION}

ML criterion, a classical parameter estimation tool, is used for state parameter estimation widely. The estimation based on ML criterion of state noise covariance $Q$ and measurement noise covariance $R$ was turned into a new measurement residual covariance $P_{\varepsilon_{k}}$ and an adaptive adjustment parameter $\alpha_{k}$ problem.

Assuming that the new measurement residual covariance $P_{\varepsilon_{k}}$ defined:

$$
P_{\varepsilon_{k}}=\frac{1}{L} \sum_{i=k-L+1}^{k} \varepsilon_{i} \varepsilon_{i}^{T}
$$

where $L$ is the size of estimate window. Hence, the objective function used to approximate the state noise estimation covariance $Q$ and the measurement noise covariance $R$ based on ML criterion defined as follows:

$$
O\left(\alpha_{k}\right)=\sum_{i=k-L+1}^{k}\left|P_{\varepsilon_{k}}\right|+\sum_{i=k-L+1}^{k} \varepsilon_{i} P_{\varepsilon_{k}}^{-1} \varepsilon_{i}^{T}
$$

where $|\cdot|$ represents the solving determinant operating, Then, carrying out derivative for objective function $O\left(\alpha_{k}\right)$, which given as follows:

$$
\frac{\partial O\left(\alpha_{k}\right)}{\partial \alpha_{k}}=\sum_{i=k-L+1}^{k}\left\{\operatorname{tr}\left[P_{\varepsilon_{k}}^{-1} \frac{\partial P_{\varepsilon_{k}}}{\partial \alpha_{k}}\right]-\varepsilon_{i}^{T} P_{\varepsilon_{k}}^{-1} \frac{\partial P_{\varepsilon_{k}}}{\partial \alpha_{k}} P_{\varepsilon_{k}}^{-1} \varepsilon_{i}\right\}
$$

where $\operatorname{tr}(\bullet)$ is the trace of matrix operating. To obtain the real time measurement noise covariance $R$, we assumed that the state noise covariance $Q$ is known, and then the Eq. (29) can be rewritten as:

$$
\sum_{i=k-L+1}^{k} \operatorname{tr}\left(P_{\varepsilon_{k}}^{-1}\left(P_{\varepsilon_{k}}-\varepsilon_{i} \varepsilon_{i}^{T}\right) P_{\varepsilon_{k}}^{-1}\right)=0
$$

due to the new measurement residual covariance $P_{\varepsilon_{k}}$,which is the positive definite matrix, in all the estimation window, the Eq. (30) always set up. So the real time state noise estimation covariance and measurement noise covariance can be obtained by following Equations, respectively.

$$
\begin{gathered}
\hat{R}_{k}^{M L}=\frac{1}{L} \sum_{i=k-L+1}^{k} \varepsilon_{i} \varepsilon_{i}^{T}-\sum_{i=0}^{2 n} W_{c}^{i}\left[y_{i}-h\left(\hat{x}_{i / k}\right)\right]\left[y_{i}-h\left(\hat{x}_{i / k}\right)\right]^{T} \\
\hat{Q}_{k}^{M L}=\frac{1}{L} \sum_{i=k-L+1}^{k}\left(G_{k} \varepsilon_{k}\right)\left(G_{k} \varepsilon_{k}\right)^{T}+P_{k}-\sum_{i=0}^{2 n} W_{c}^{i}\left(\chi_{k / k-1}^{i}-\hat{x}_{k / k-1}\right)\left(\chi_{k / k-1}^{i}-\hat{x}_{k / k-1}\right)^{T}
\end{gathered}
$$

\subsection{HYBRID ADAPTIVE UKF}

With the prior statistical characteristics of the noise were unknown, the estimation covariance of the noise may be deviate from the real value that leads to the increase of the state estimation error, which is the fundamental reason resulting in divergence of the filtering. UKF method uses the Kalman filtering framework. However, Kalman filtering is a growth of 
memory filtering. At the initial stage of the filter, the updated covariance $P_{k-1 / k-1}$ is larger relatively, hence, it is important to take full use of the measurement information which has a stronger correction ability to make the estimation close to truth value fast. Unfortunately, with the advance of the filtering process, $P_{k-1 / k-1}$ is gradually reduced, and the gain of the filtering process is getting smaller and smaller, so that the correction ability of the new measurement data for the real state become more and more little, which may be arouse the divergence of the filtering.

Whether the UKF based MAP or based ML criterion, the finally goal is to obtain the noise state covariance $Q$ and the measurement noise covariance $R$, which closes to or converges to the real value. A suboptimal unbiased estimator based on MAP, which sometimes maybe arouse fluctuations under the correction amplitude controlled perishing. Nevertheless, the UKF based on ML, which tunes the noise estimation into solving the expectation maximization of a log likelihood function, which may fall into local optimum. In order to improve the stability of UKF, with unknown which the noise state covariance $Q$ and the measurement noise covariance $R$ from the method based on MAP and ML, respectively, is more close to the truth value.

Assuming that the state noise covariance estimation and the measurement noise estimation obtained by MAP and ML, defined as $\hat{Q}_{k}^{M A P}, \hat{R}_{k}^{M A P}$ and $\hat{Q}_{k}^{M L}, \hat{R}_{k}^{M L}$ respectively. In order to reduce the influence for the filter on the stability, which arouse from estimation based MAP and ML. The mean value of $\hat{Q}_{k}^{M A P}, \hat{Q}_{k}^{M L}$ and $\hat{R}_{k}^{M A P}, \hat{R}_{k}^{M L}$ can obtain by the following equations:

$$
\begin{gathered}
\hat{Q}_{k}^{N e \underline{\underline{w}}} \frac{\hat{Q}_{k}^{M A P}+\hat{Q}_{k}^{M L}}{2} \\
\hat{R}_{k}^{\text {New }}=\frac{\hat{R}_{k}^{M A P}+\hat{R}_{k}^{M L}}{2}
\end{gathered}
$$

When the following condition Equations were satisfied, which defined as follows, we looks the filter stability and convergence:

$$
\begin{aligned}
& \frac{1}{k} \sum_{i=1}^{k}\left(\hat{Q}_{i}^{N e w}-\hat{Q}_{i-1}^{N e w}\right)<\Delta \zeta \\
& \frac{1}{k} \sum_{i=1}^{k}\left(\hat{R}_{i}^{N e w}-\hat{R}_{i-1}^{N e w}\right)<\Delta \zeta
\end{aligned}
$$

where $\Delta \zeta$ is a pre-given very small positive integer. And then, the whole algorithm of this proposed is introduced detailed as below.

\section{Algorithm of this proposed}

Step1: Equation (4) to Eq. (15) used to calculate the convention UKF method;

Step2: Equation (22) and Eq. (23) employed to obtain the state noise mean and measurement noise mean, respectively;

Step3: Equation (25) and Eq. (24) used to compute the state noise covariance $\hat{Q}_{k}^{M A P}$ and measure covariance $\hat{R}_{k}^{M A P}$, Eq. (32) and Eq. (31) are used for getting the state noise covariance $\hat{Q}_{k}^{M L}$ and measure covariance $\hat{R}_{k}^{M L}$; 
Step4: Equation (33) and Eq. (34), are used to compute the state noise covariance $\hat{Q}_{k}^{\text {New }}$ and measure covariance $\hat{R}_{k}^{\text {New }}$, at the same time, the Eq. (35) and Eq. (36) employed to judge the stability of the filter, if the filter is not convergence then go to Step2;

Step5: Finally, the estimation results from Step2, Step3 and Step4 are sent to the Step1 for obtaining the corresponding UKF.

\section{SIMULATION AND CASE STUDY}

In this section, we provide a practical case study to compare the performance with the method presented in [16-17]. Subsequently, the results from simulation are analysed.

A strongly nonlinear Gaussian system model, which described as follows, employed to verify the effectiveness of this proposed method:

$$
\left\{\begin{array}{l}
x_{k}=\frac{1}{2} x_{k-1}+\frac{0.2 x_{k-1}}{1+x_{k-1}^{2}}+q_{k-1} \\
y_{k}=10 x_{k}+\frac{1}{20} x_{k}^{2}+r_{k}
\end{array}\right.
$$

where $q_{k-1}$ and $r_{k}$ are the white Gaussian noise, and the statistical properties of constant values defined as follows:

$$
\begin{cases}q=1.2, & r=1.0 \\ Q=0.6, & R=0.8\end{cases}
$$

Assumed that the initial value of the nonlinear system given as:

$$
\left\{\begin{array}{l}
\hat{x}_{0}=1.1 \\
P_{0}=0.01
\end{array}\right.
$$

$\hat{x}_{0}, q_{k-1}$ and $r_{k}$ are not related to each other. And the state noise statistics characteristics are unknown, the initial state noise estimation mean and covariance presents as below:

$$
\left\{\begin{array}{l}
\hat{q}_{0}=0.2, \\
\hat{Q}_{0}=0.5
\end{array}\right.
$$

In order to compare the performance of all kinds of UKF conveniently, the method introduced in [16] named Lin's UKF and the method reported in[17] described as Wang's UKF, respectively. And then, the state noise estimation covariance $\hat{Q}$ can be obtained by using these methods mentioned afore, the results of each method used to get the state noise estimation covariance given as following Figure s detailed. 


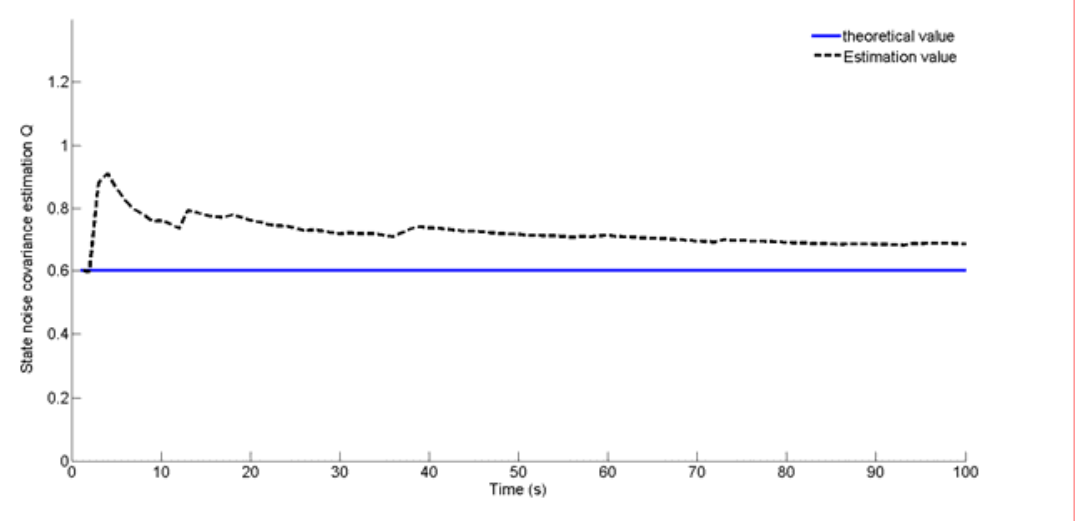

Fig. 1 State estimation covariance of Lin's UKF

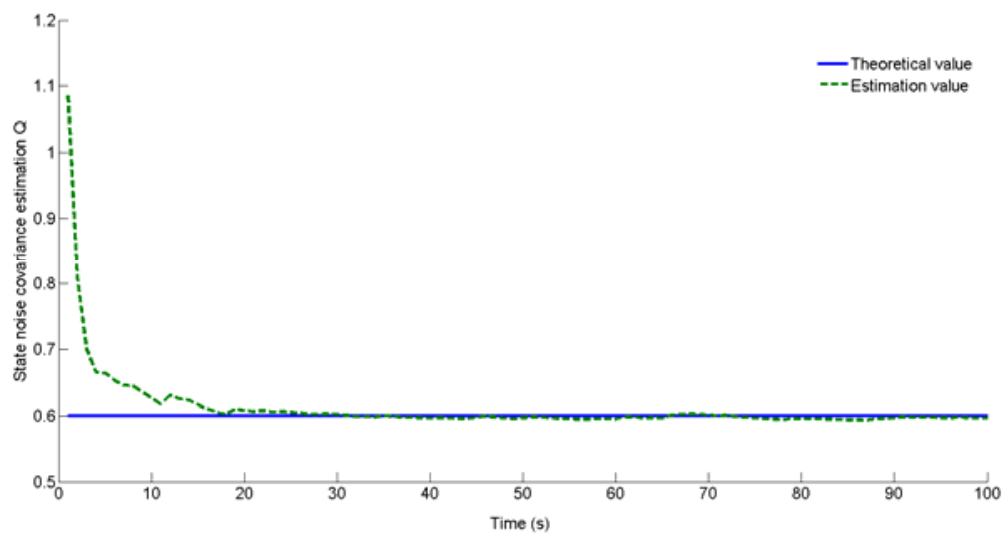

Fig. 2 State estimation covariance of our UKF

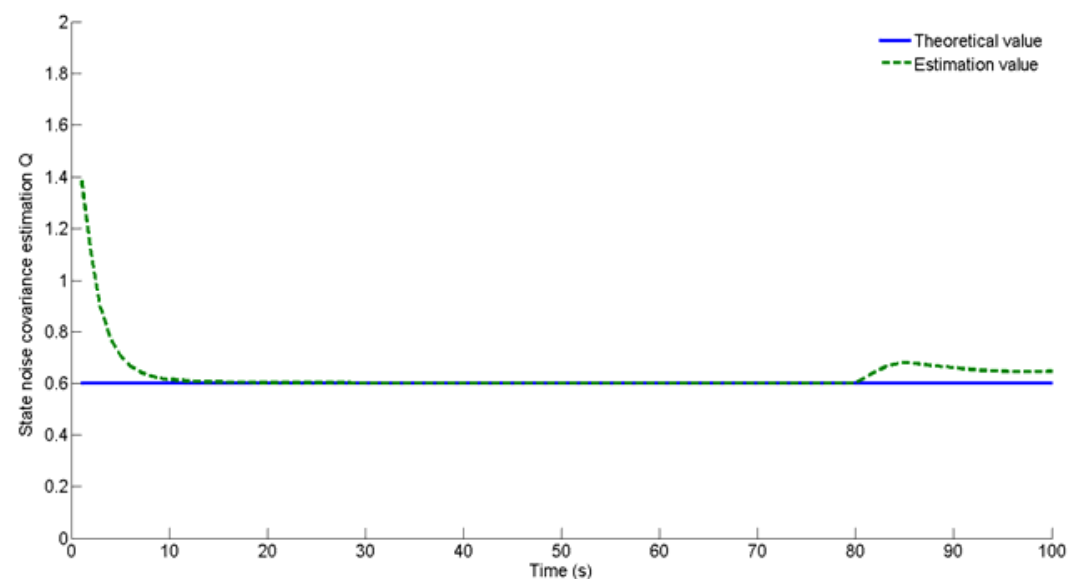

Fig. 3 State estimation covariance of Wang's UKF

Illustrated in Figure 1, Figure 2 and Figure 3, the convergence speed of the state noise estimation covariance of Lin's UKF is slower than that of the other, which has a defect is when 
convergence to the optimal value it begins to diverge. The main reason which leads to this problem is the inappropriate fading factor adjustment maybe enhance or slacking down the effect for the maximum a posteriori based UKF method in error. Figure 3 shows the Wang's UKF can quickly converge, however, as time goes on, there is a suddenly diverge appearance and then slowly converge. The ML and EM based UKF can convergence fast, but it exists over fitting defect, which leads to burst diverge. The performance of our UKF for state noise estimation covariance shows in Figure 2, the convergence speed of our UKF is between Lin's UKF and Wang's UKF. Nevertheless, in all the computing process, the state noise estimation covariance can converge to the theoretical value stability. Due to in the estimation process, the mean of state noise estimation covariance of Lin's and Wang's is computed, which can avoid the defects from a single method.

On the basis of the above model, assuming that the state noise $q$ and measurement noise $r$ are Gaussian noise and obey Gauss distribution, the state noise statistics characteristic define as Eq. (40), however, the measurement noise statistics characteristic are unknown, but the initial value of measurement noise statistics characteristic represented as follows.

$$
\left\{\begin{array}{l}
\hat{r}_{0}=0.3 \\
\hat{R}_{0}=0.6
\end{array}\right.
$$

The simulation results illustrate as Figure 4, Figure 5 and Figure 6.

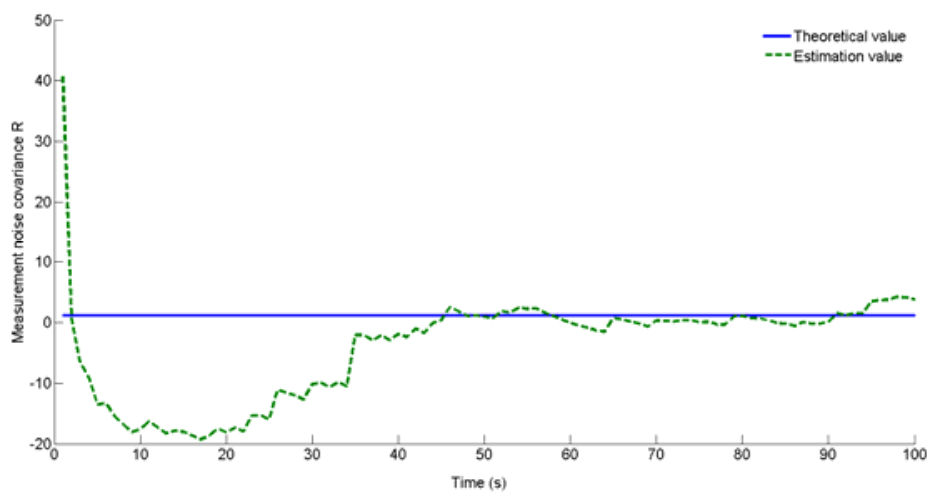

Fig. 4 Measurement noise covariance of Lin's UKF

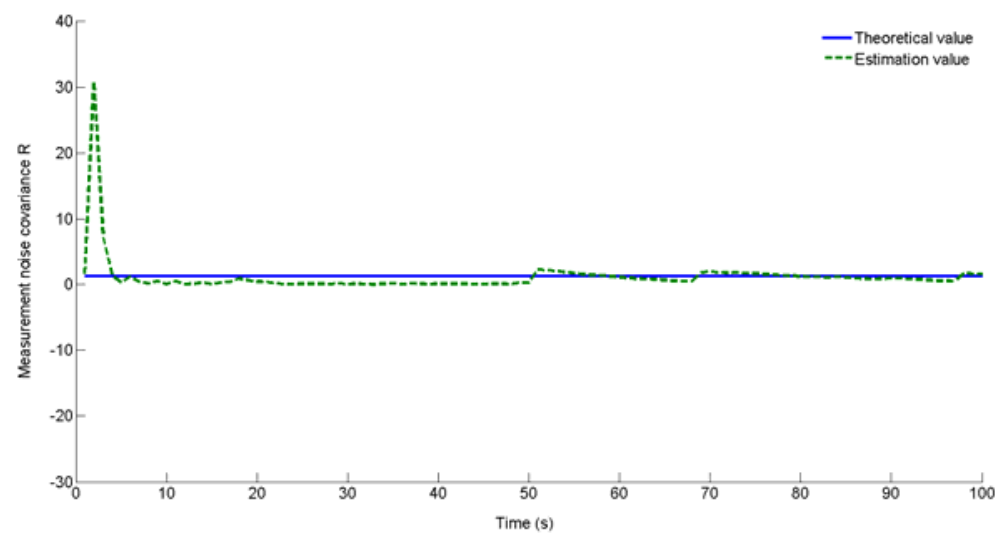

Fig. 5 Measurement noise covariance of our UKF 


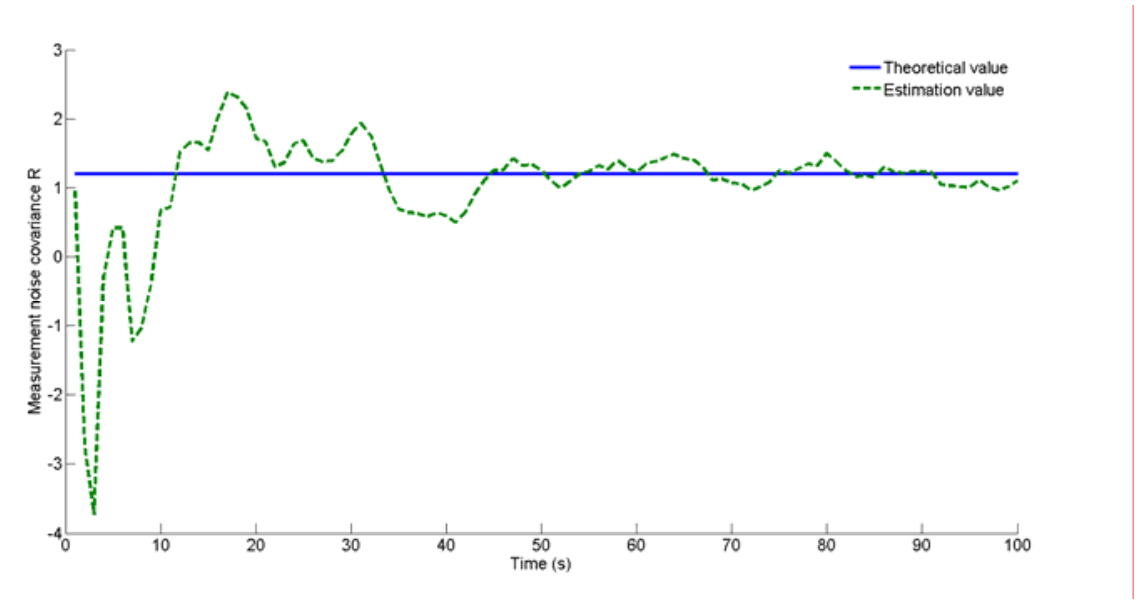

Fig. 6 Measurement noise covariance of Wang's UKF

Illustrated in Figure 4, Figure 5 and Figure 6, at initial phase, due to lack of prior knowledge and the fading factor adjusting, the measurement noise covariance estimated by Lin's UKF has large fluctuations, and then it can convergence to the theoretical value. Described as Figure 6, in the initial phase, the estimation of measurement noise covariance has smaller fluctuations than that of the other's. As the filter deepen, estimation of measurement noise covariance can convergence to the theoretical value accurately. Owing to in the filter process, our UKF makes good useful of the advantages of the Lin's UKF and Wang's UKF which the mean of measurement noise covariance from Lin's UKF and Wang's UKF used to a new UKF iteration.

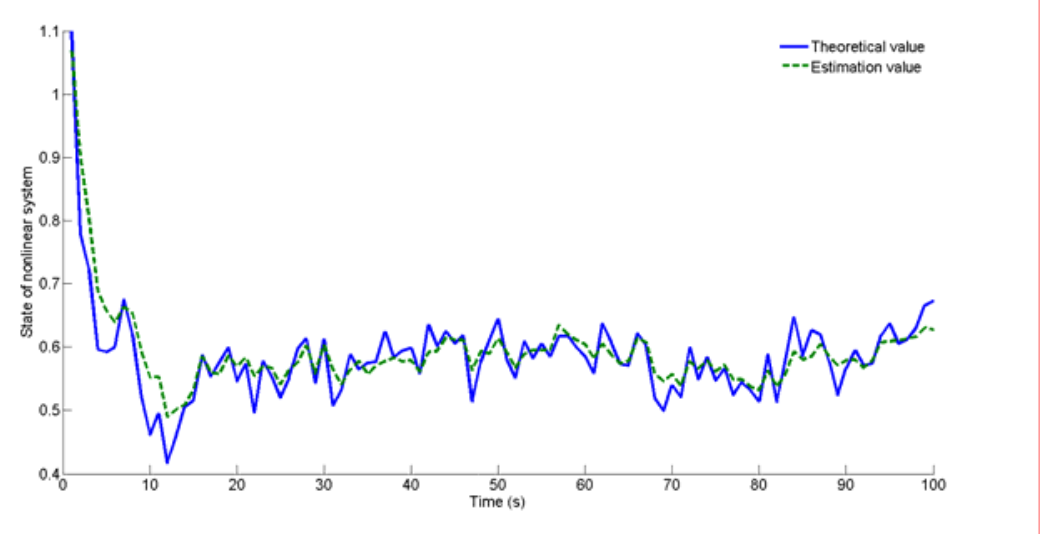

Fig. 7 Performance of Lin's UKF 


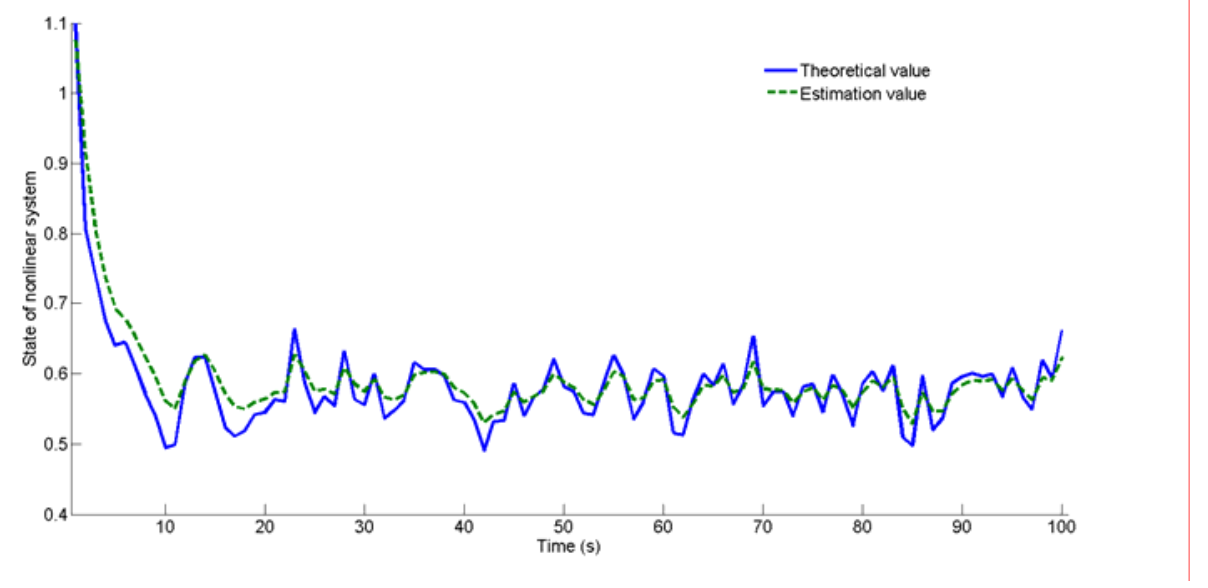

Fig. 8 Performance of our UKF

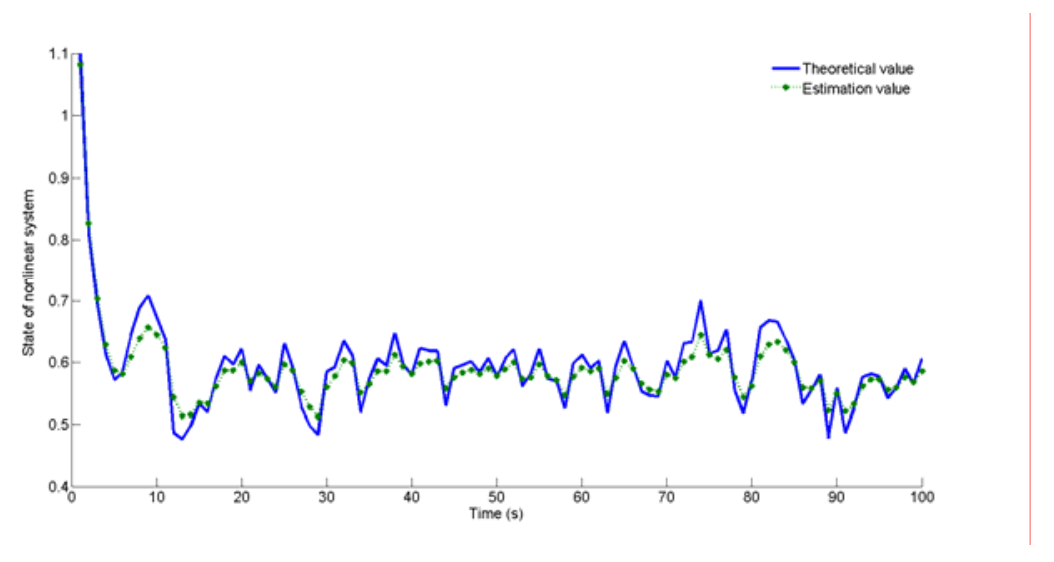

Fig. 9 Performance of Wang's UKF

From the Figure 7, Figure 8 and Figure 9, it is very obviously to know that the performance of our UKF is better than the other two methods. In all the filter process, the state of nonlinear system, which can approximation to theoretical value.

\section{CONCLUSIONS}

From the study of the adaptive unscented Kalman filter, it is very important to do best on improving the estimation covariance of state noise and measurement noise precision. In this paper, we introduce a hybrid adaptive UKF method for nonlinear system state estimation, where the UKF based MAP and ML used to compute the estimation covariance of state noise and measurement noise, respectively, while the hybrid adaptive UKF makes the estimation covariance of state noise and measurement noise of the innovation as small as possible. The basic idea behind our UKF method is to build an balance operation to obtain a closer optimum estimation. And then employ the convergence strategy, to accomplish the filter stability decide. Convergence analysis shows that the proposed method is better than the Lin's and Wang's method, respectively. 


\section{ACKNOWLEDGMENTS}

This work was supported by the National Natural Science Foundation of China (Grant: 61301300, 61673400), Guangdong Natural Science Foundation (Grant: 2016A030313823).

\section{REFERENCES}

[1] F. Auger, et al., Industrial Applications of the Kalman Filter: A Review, IEEE Transactions on Industrial Electronics, Vol. 60, pp. 5458-5471, 2013.

https://doi.org/10.1109/TIE.2012.2236994

[2] C.D. Zuluaga, et al., Short-term wind speed prediction based on robust Kalman filtering: An experimental comparison, Applied Energy, Vol. 156, pp. 321-330, 2015.

https://doi.org/10.1016/j.apenergy.2015.07.043

[3] L. Salvatore, et al., A new EKF-based algorithm for flux estimation in induction machines, IEEE Transactions on Industrial Electronics, Vol. 40, pp. 496-504, 1993.

https://doi.org/10.1109/41.238018

[4] K. Jiang, et al., An extended Kalman filter for input estimations in diesel-engine selective catalytic reduction applications, Neurocomputing, Vol. 171, pp. 569-575, 2016.

https://doi.org/10.1016/j.neucom.2015.06.072

[5] F. Meurer, et al., Nonlinear state estimation for the Czochralski process based on the weighing signal using an extended Kalman filter, Journal of Crystal Growth, Vol. 419, pp. 57-63, 2015. https://doi.org/10.1016/j.jcrysgro.2015.02.099

[6] J.A. Delgado-Aguiñaga, et al., Multi-leak diagnosis in pipelines based on Extended Kalman Filter, Control Engineering Practice, Vol. 49, pp. 139-148, 2015.

https://doi.org/10.1016/j.conengprac.2015.10.008

[7] S.J. Julier, et al., A new approach for filtering nonlinear systems, in Proceedings of the American Control Conference, Vol. 3, pp. 1628-1632, 1995.

https://doi.org/10.1109/ACC.1995.529783

[8] S. Julier, et al., A new method for the nonlinear transformation of means and covariances in filters and estimators, IEEE Transactions on Automatic Control, Vol. 45, pp. 477-482, 2000. https://doi.org/10.1109/9.847726

[9] Q. Song and J.-D. Han, An Adaptive UKF Algorithm for the State and Parameter Estimations of a Mobile Robot, Acta Automatica Sinica, Vol. 34, pp. 72-79, 2008.

https://doi.org/10.3724/SP.J.1004.2008.00072

[10] M.T. Sabet, et al., Extended and Unscented Kalman filters for parameter estimation of an autonomous underwater vehicle, Ocean Engineering, Vol. 91, pp. 329-339, 2014.

https://doi.org/10.1016/j.oceaneng.2014.09.013

[11] A.M. Khoshnood and O. Kavianipour, Vibration Suppression of Fuel Sloshing using Subband Adaptive Filtering, International Journal of Engineering, TRANSACTIONS A: Basics, Vol. 28, pp. 1507-1514, 2015. 
[12] M. Partovibakhsh and L. Guangjun, An Adaptive Unscented Kalman Filtering Approach for Online Estimation of Model Parameters and State-of-Charge of Lithium-Ion Batteries for Autonomous Mobile Robots, IEEE Transactions on Control Systems Technology, Vol. 23, pp. 357-363, 2015. https://doi.org/10.1109/TCST.2014.2317781

[13] H. He, et al., Real-time estimation of battery state-of-charge with unscented Kalman filter and RTOS $\mu$ COS-II platform, Applied Energy, Vol. 162, pp. 1410-1418, 2016. https://doi.org/10.1016/j.apenergy.2015.01.120

[14] C. Liu, et al., Modified unscented Kalman filter using modified filter gain and variance scale factor for highly maneuvering target tracking, Journal of Systems Engineering and Electronics, Vol. 25, pp. 380-385, 2014. https://doi.org/10.1109/JSEE.2014.00043

[15] C. Liu, et al., Unscented extended Kalman filter for target tracking, Journal of Systems Engineering and Electronics, Vol. 22, pp. 188-192, 2011. https://doi.org/10.3969/j.issn.1004-4132.2011.02.002

[16] Z. Lin, et al., Adaptive UKF Filtering Algorithm Based on Maximum a Posterior Estimation and Exponential Weighting, Acta Automatica Sinica, Vol. 36, pp. 1007-1019, 2010. https://doi.org/10.3724/SP.J.1004.2010.01007

[17] L. Wang, et al., An adaptive UKF algorithm based on maximum likelihood principle and expectation maximization algorithm, Acta Automatica Sinica, Vol. 38, pp. 1200-1210, 2012. https://doi.org/10.3724/SP.J.1004.2012.01200

[18] F. Deng, et al., Adaptive unscented Kalman filter for parameter and state estimation of nonlinear high-speed objects, Journal of Systems Engineering and Electronics, Vol. 24, pp. 655-665, 2013. https://doi.org/10.1109/JSEE.2013.00076

[19] H.X. Le and S. Matunaga, A residual based adaptive unscented Kalman filter for fault recovery in attitude determination system of microsatellites, Acta Astronautica, Vol. 105, pp. 30-39, 2014. https://doi.org/10.1016/j.actaastro.2014.08.020

[20] W. Zhang, et al., Adaptive unscented Kalman filter based state of energy and power capability estimation approach for lithium-ion battery, Journal of Power Sources, Vol. 289, pp. 50-62, 2015. https://doi.org/10.1016/j.jpowsour.2015.04.148

[21] W. Li, et al., Robust unscented Kalman filter with adaptation of process and measurement noise covariances, Digital Signal Processing, Vol. 48, pp. 93-103, 2016. https://doi.org/10.1016/j.dsp.2015.09.004

[22] W. Li, et al., Windowing-based adaptive unscented Kalman filter for spacecraft relative navigation, in Control Conference (CCC), 2015 34th Chinese, pp. 5136-5141, 2015.

[23] S. Gao, et al., Windowing and random weighting-based adaptive unscented Kalman filter, International Journal of Adaptive Control and Signal Processing, Vol. 29, pp. 201-223, 2015. https://doi.org/10.1002/acs.2467

[24] L. Yu, et al., UKF Based Nonlinear Filtering Using Minimum Entropy Criterion, IEEE Transactions on Signal Processing, Vol. 61, pp. 4988-4999, 2013.

https://doi.org/10.1109/TSP.2013.2274956

[25] A. Sage and G.W. Husa, Adaptive filtering with unknown prior statistics, in Proceeding of Joint Automatic Control Conference, pp. 760-769, 1969. 